\section{Commentary: Getting in the zone: Thoracic endovascular aortic repair safety in Ishimaru zones 0 and 1}

\author{
Evan P. Rotar, MD, MS, and Irving L. Kron, MD
}

Patients with aortic arch aneurysms are a unique group at high risk due to the natural history of their pathology and the requisite interventions needed to treat the disease. The mainstay of treatment has long been open surgical repair, although this paradigm is changing. The advent and progression of thoracic endovascular aortic repair (TEVAR) has expanded treatment options for high-risk surgical patients and those with complicated aneurysm architecture. Dake and colleagues ${ }^{1}$ are to be commended for a thoughtful and well-articulated article describing their early and late outcomes of using a novel, branched aortic endograft for aortic arch aneurysms in high-risk patients.

The primary endpoints of this multicenter, prospective, nonrandomized feasibility study were successful deployment of the endograft and patency of the branch vessel on procedure completion, both of which were successful in all patients. Endovascular treatment of Ishimaru zone 0 and 1 aorta necessitates supra-aortic revascularization, and in this group, no neurologic events were identified after any such intervention before TEVAR despite differing strategies. There was no early (30-day) mortality in the cohort; however, 2 patients (22\%) did meet the World Health Organization (WHO) criteria for stroke after TEVAR. Furthermore, the length of postoperative hospital stay was

\footnotetext{
From the Division of Thoracic and Cardiovascular Surgery, Department of Surgery, University of Virginia, Charlottesville, Va.

Disclosures: The authors reported no conflicts of interest.

The Journal policy requires editors and reviewers to disclose conflicts of interest and to decline handling or reviewing manuscripts for which they may have a conflict of interest. The editors and reviewers of this article have no conflicts of interest.

This work was supported by a research grant from the National Institutes of Health, National Heart, Lung, and Blood Institute (T32HL007849). The content is solely the responsibility of the authors and does not represent the official views of the National Institutes of Health.

Received for publication Jan 30, 2021; revisions received Jan 30, 2021; accepted for publication Feb 1, 2021; available ahead of print Feb 3, 2021.

Address for reprints: Irving L. Kron, MD, Division of Thoracic and Cardiovascular Surgery, Department of Surgery, University of Virginia, 1215 Lee St, PO Box 800679, Charlottesville, VA 22908 (E-mail: ILK@virginia.edu).

JTCVS Techniques 2021;7:7-8

2666-2507

Copyright (C) 2021 The Authors. Published by Elsevier Inc. on behalf of The American Association for Thoracic Surgery. This is an open access article under the CC BY-NCND license (http://creativecommons.org/licenses/by-nc-nd/4.0/).

https://doi.org/10.1016/j.xjtc.2021.02.001
}

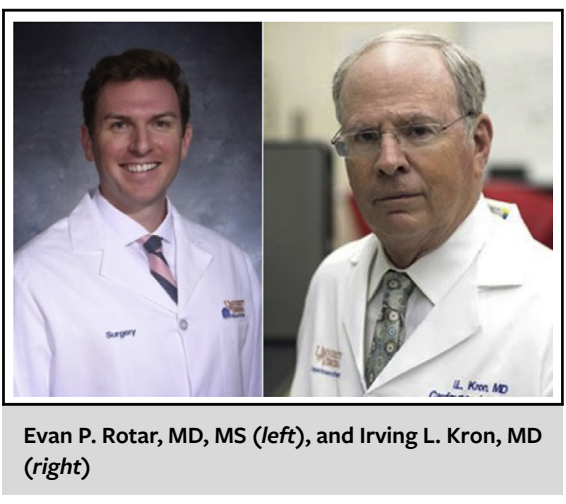

CENTRAL MESSAGE

Single-branch endovascular treatment of Ishimaru zone $\mathrm{O}$ and 1 aortic arch aneurysms can be performed with acceptable early safety in appropriately selected patients.

variable among the cohort, ranging from 3 to 48 days (median, 20 days). It would be worthwhile to know whether any perioperative factors impacted this variability, especially because the median is slightly longer than that previously reported for hybrid repairs. ${ }^{2}$

With respect to 12-month outcomes, 2 patients died, 1 of whom sustained a periprocedural stroke, although their death events were deemed unrelated to the index procedure. The other periprocedural stroke patient experienced a bilateral frontal lobe and left parietal lobe stroke at 6 months, although whether this was related to the device was unknown. No surviving patients demonstrated evidence of aneurysm growth; however, 3 people exhibited evidence of endoleak at 1 year. There was no mention of whether these patients required treatment of the endoleak. These findings reinforce that although the technical feasibility of the index operation is important, so are the long-term safety of these patients.

Dake and colleagues previously reported the successful use of this single branch endograft treatment of zone 2 disease, ${ }^{3}$ and they further expand on its utility with the present investigation. They provide compelling evidence that patients with complex proximal aortic arch disease can undergo treatment with single-branch TEVAR in Ishimaru zone 0 or 1 and achieve acceptable levels of safety in the early postsurgical period. Although these interventions can be performed with acceptable early survival, we must 
keep in mind that these are high-risk patients with the potential for significant complications, especially when the dependency of all arch vessel territories rely on a single vascular supply. The authors' acknowledgment of validated stroke scales cannot be overstated, and these should be implemented in future investigations. Ultimately, this important work reinforces that although results are mixed, this is just the beginning for the final frontier in aortic endografting.

\section{References}

1. Dake MD, Bavaria JE, Singh MJ, Oderich G, Filinger M, Fischbein MP, et al. Management of arch aneurysms with a single-branch thoracic endograft in zone 0. J Thorac Cardiovasc Surg Tech. 2021;7:1-6.

2. Vallabhajosyula P, Szeto W, Desai N, Bavaria JE. Type I and type II hybrid aortic arch replacement: postoperative and mid-term outcome analysis. Ann Cardiothorac Surg. 2013;2:280-7.

3. Patel HJ, Dake MD, Bavaria JE, Singh MJ, Filinger M, Fischbein MP, et al. Branched endovascular therapy of the distal aortic arch: preliminary results of the feasibility multicenter trial of the Gore thoracic branch endoprosthesis. Ann Thorac Surg. 2016;102:1190-8. 